\title{
Research on Equipment Management System Based on Robot Laboratory
}

\author{
Chong Hu', Xin Wang1, Yunhui Wang² \\ ${ }^{1}$ School of Data Science and Software Engineering, Baoding University, Baoding, China \\ ${ }^{2}$ School of Automotive and Electronic Engineering, Baoding University, Baoding, China \\ Email: wangxincloud@163.com
}

How to cite this paper: $\mathrm{Hu}, \mathrm{C}$., Wang, $\mathrm{X}$. and Wang, Y.H. (2020) Research on Equipment Management System Based on Robot Laboratory. Journal of Computer and Communications, 8, 23-31. https://doi.org/10.4236/jcc.2020.87003

Received: July 2, 2020

Accepted: July 20, 2020

Published: July 23, 2020

Copyright () 2020 by author(s) and Scientific Research Publishing Inc. This work is licensed under the Creative Commons Attribution International License (CC BY 4.0).

http://creativecommons.org/licenses/by/4.0/

(c) (i) Open Access

\begin{abstract}
With the expansion of the scale of our hospital robot laboratory, equipment use, reserves are increasing, manual management equipment information has been unable to adapt to daily management, such as data record error rate increased, the number of experiments increased, equipment loss and maintenance is not clearly defined. In order to ensure the safety and accuracy of the equipment, a system of equipment management systems was developed as a result of the conversion to the use of management systems for the recording and storage of information. The design of this system is a perfect robot laboratory equipment management system based on the development of web framework technology for equipment management functions. The back end of this system uses the Java language, the SSM framework as the main body, the database is built by MySQL relational database, the front-end page uses JSP, Bootstrap, jQuery and other technologies, and uses Eclipse project construction tools for system development. After a period of trial operation, the level of laboratory equipment management has been significantly improved.
\end{abstract}

\section{Keywords}

Device Management, Java, Spring, SpringMVC, MyBatis

\section{Introduction}

With the expansion of teaching scale and changes in teaching methods, there are more and more practical courses in the robot laboratory, which will inevitably be accompanied by the expansion of the laboratory and the increase of equipment. Management method, it is difficult to comprehensively and carefully manage the equipment; the storage and use of equipment are often not documented and the time of use is defined; there is no relevant record and maintenance of equipment 
damage. At present, most of the equipment information management is lacking in the integration of equipment information management, maintenance management, borrowing management, and the three-in-one refined management. Therefore, in order to improve the management method of the laboratory, it is urgent to start the development of a laboratory equipment management system for business process innovation [1].

The implementation of this system will effectively manage the information of the equipment to improve the utilization rate of the equipment and the work efficiency of the laboratory members; the sound borrowing management will also avoid unwritten verbal borrowing to guarantee the safety of the equipment; the accurate category management will be more detailed the classified equipment can efficiently locate related categories through category query and insert new equipment in the future to improve management efficiency; regular maintenance and repair can be immediately reported, and the equipment can be repaired and replaced, effectively reducing equipment reuse defects such as low rate and long-term shutdown [2]. This system transforms multi-dimensional unified and scientific management equipment to realize the whole process management of equipment, from extensive management to fine management.

\section{System Analysis}

Equipment information management function: This function contains information of all equipment in the laboratory. When entering as an administrator, the administrator implements operations such as adding, deleting, modifying, and querying device information. When students enter as a user, they only have functions such as viewing and borrowing [3].

Equipment category management function: This function contains the category information of all equipment in the laboratory. The administrator classifies the equipment in the laboratory equipment uniformly. It mainly performs functions such as query, modification, deletion, and addition of device category information.

Administrator information management and student information management function: This function records the data of all members in the laboratory, including administrator information and student information, divided into administrator and student modules. Administrators can register new accounts for students or new administrators, or add or delete account-related information, such as the addition of accounts and the modification of passwords.

Equipment maintenance management function: This function is based on the user's identity information, the treatment of equipment for repair is also different, so it is divided into two modules. The administrator in the repair module is only responsible for reviewing and processing the equipment to be repaired that has been applied for repair or replacement. Student users can add a repair application to the repair equipment, query the information they have repaired, or modify the corresponding repair type and other functions [4]. 
Equipment borrowing management function: This function is divided into three modules: lending, returning and querying. Among them, borrowing is to query the borrowed information and add borrowing records. Returning refers to querying the returned information and adding return records. To review and process borrowed or returned information. Student users can borrow and return. Among them, borrowing mainly refers to querying borrowable equipment, submitting borrowing, and querying already borrowed equipment, while returning mainly refers to price-increasing return and querying already returned equipment.

Borrowing duration management function: the purpose of this function is to set the maximum borrowing duration and maximum borrowing quantity, and control the borrowing scope.

\section{Technical Introduction}

The system is developed on the Eclipse platform, using the SSM framework, which is based on the extension of SpringMVC from Spring, plus the technical framework combined with MyBatis, the system uses the SSM framework as the system main framework to build the system.

Spring's IOC (Control Inversion) greatly reduces the coupling of code, better reuse and extension procedures, and AOP (Aspect-Oriented Programming), through AOP cuts the repeated code, cutting some repetitive sub-processes into a horizontal. After cutting, a horizontal surface is formed, that is, the cut surface. The separated module exists alone and can be used at any time without destroying the original class. The module can add new methods and functions to achieve improvement and enhancement its role.

The development model of SpringMVC is to communicate with the database through the model layer, and respond to and process http requests from the client through the Controller layer. After processing and returning the desired content, this part of the content is rendered and parsed by the view layer. Therefore, only SpringMVC cannot support a powerful and efficient page. It also needs to interact with Spring. Because Spring is responsible for the coupling of the control layer and the persistence layer, it can operate on the configuration of the database and cache, but SpringMVC cannot be implemented. Therefore, if you want to use SpringMVC, you must rely on Spring. Spring and SpringMVC are inseparable.

MyBatis is a persistence layer framework, which encapsulates JDBC, so that developers do not need to write a lot of repeated JDBC code. The mechanism of object-relational mapping provided by MyBatis allows developers to configure the mapping only by writing XML files or using annotations, so that object-relational mapping can be established between the entity class and the database, and developers can only dynamically in the xml configuration file without modifying the code. You can modify the SQL statement, so that the non-intrusive modification releases the coupling of SQL and code, which greatly facilitates the operation of developers [5]. 
Bootstrap integrates three technical achievements of HTML, CSS, and JavaScript, making it an excellent front-end framework with a powerful class library, excellent compatibility, and convenient operation. It encapsulates a variety of components, styles and plug-ins. When you use it, you can build a fully functional, extensible, neatly styled and simple style website by simply introducing a link. This development method will be a kind of development. Innovation has greatly improved the development efficiency of developers.

SSM is a framework integrated with Spring framework, SpringMVC framework and Mybatis framework. The following is a schematic diagram of SSM, showing the flow of program execution and the connections within the framework, as shown in Figure 1.

\section{System Design}

The overall function of the system is composed of equipment maintenance management, equipment borrowing management, equipment information management, administrator information management, student information management, and borrowing duration management.

Device information management design: When the user enters the device information on the client and sends a new request, the database will receive the relevant data, store the information in the library, and delete and modify the operation. When the user performs a request to view or find a device, the database retrieves the data and returns it to the page [6].

Administrator information management design: Only administrators perform requests to add, delete, and modify user information. After the request is sent, it must be stored in the database, and it can be taken out for display. The design of student information management is the same.

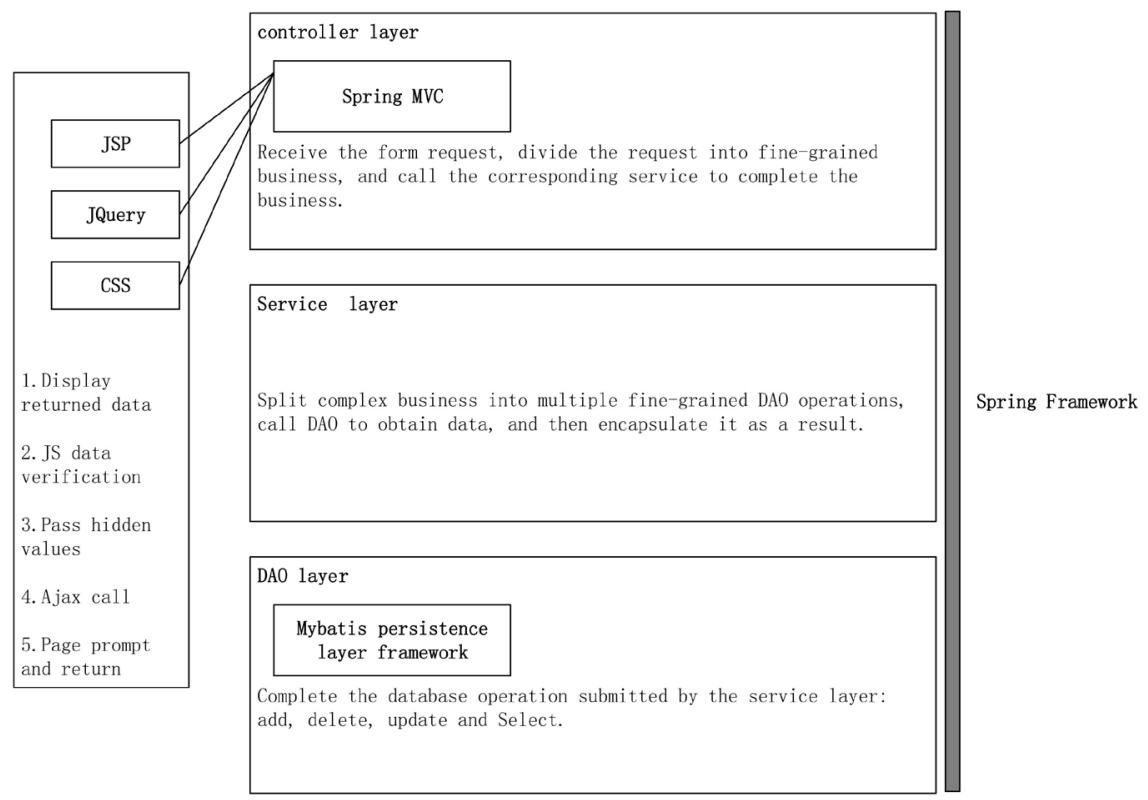

Figure 1. Schematic diagram of SSM framework. 
Equipment maintenance management design: The user needs to choose to add related equipment for repair. After the repair request is completed, the repair information will be recorded in the database, and the user can view the relevant information.

Design of equipment borrowing management: Submit a request for equipment borrowing or return in the system, save the information in the database, and then return to the administrator for review and processing the timing diagram of equipment borrowing management is shown in Figure 2.

Borrowing duration management design: The administrator sets the borrowing duration and the number of requests, the database records the setting information, and then returns the results for corresponding processing.

The system is divided into two kinds of permissions, administrator and student. When the user enters the system, the identity is selected according to their account permissions. After login verification, the account and password are confirmed before entering the system. Log in with different identities will enter different pages. The corresponding functions of different pages are different, so you can selectively use each function module according to your needs. The following is an example of an administrator business process diagram, as shown in Figure 3.

The system uses the MySQL relational database to divide the relevant attributes. According to the equipment and user information and the return of the equipment, type, maintenance, borrowing time and other operations, the attributes of each function are divided and related the attributes are divided into tables, so 7 data tables are designed.

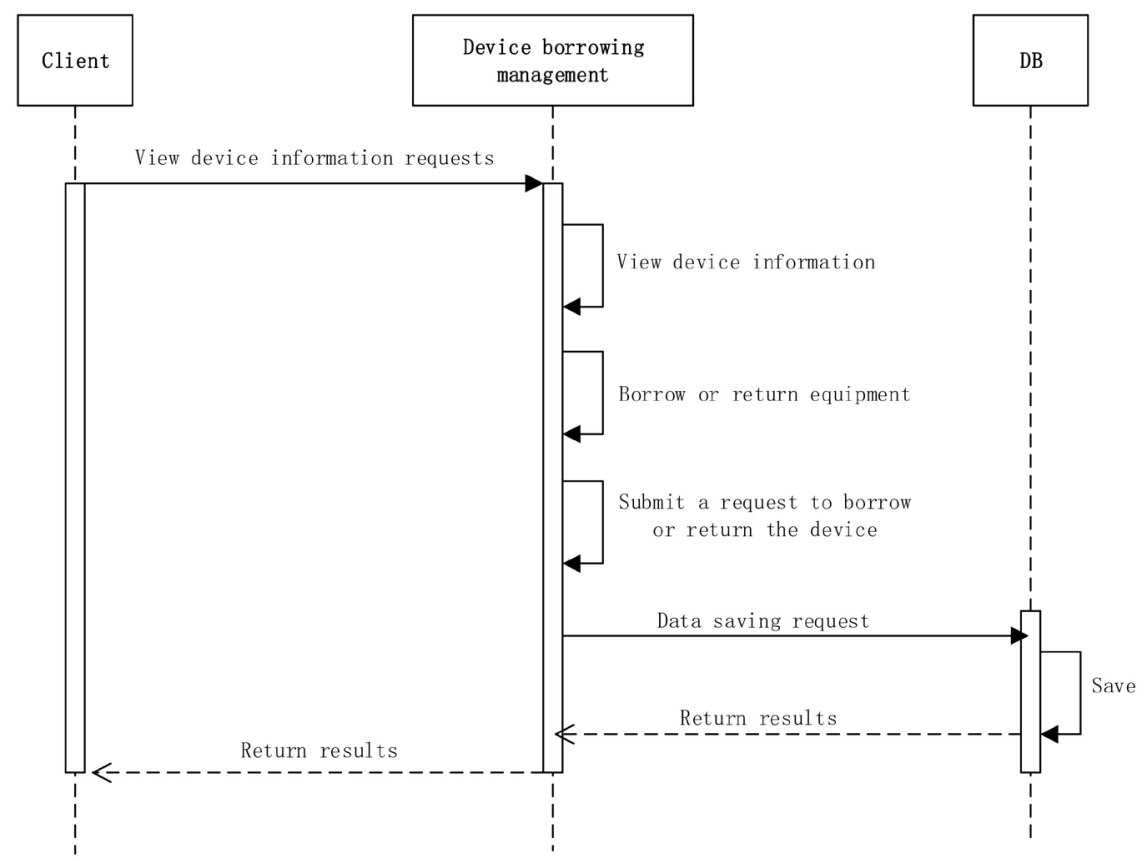

Figure 2. Timing diagram of equipment borrowing management. 


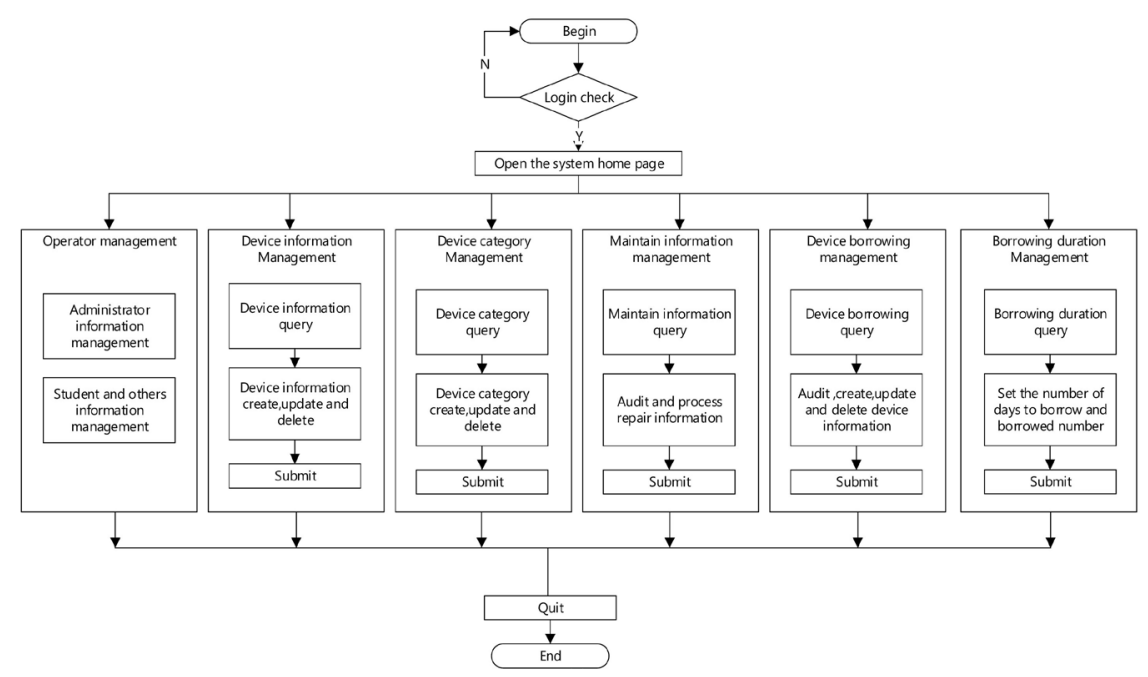

Figure 3. Administrator business flow chart.

Administrator information table: The name of the table is robot_admin, and the account, name, and password are stored in the table, and the administrator can use the permissions of the administrator through the account of the administrator [7].

Student information table: The table is named robot_student, and the student ID, name, and password are stored, and the role is similar to the administrator.

Borrowing time table: The table is named robot_setting, and the table contains the properties of borrowing time and borrowing quantity.

Device information table: This table is named robot_devices, and this table stores the relevant attributes of laboratory equipment.

Device category table: This table is named robot_devices_type, and this table sets the category attribute of the device.

Equipment repair form: The form is named robot_devices_wrong_student, and the form covers the information after the student repairs the equipment.

Device borrowing table: This table is named robot_devices_student and stores all users' borrowing information.

\section{System Implementation}

\subsection{Login Module}

Before entering the system, you need to enter the accurate password and account number. If the input method is incorrect, the front desk will return an error response requesting the user to enter the account information correctly; the input format is correct, you can submit the information, the system will log in to verify and connect The database inquires whether the account is correct, and can only enter the system after passing the verification, otherwise it is necessary to return to the login page to log in again. In order to ensure the security of the system, it is also necessary to set up a login interceptor to intercept the illegal login to the system. 


\subsection{Device Information Management Module}

This module displays various equipment information in the laboratory. In addition to viewing all information, the administrator can also add newly purchased equipment and delete damaged equipment information in the laboratory.

\subsection{Administrator Information Management and Student Information Management Module}

This module is used by the administrator. When the person in charge of the laboratory changes, the administrator information needs to be changed. Here, the administrator module can be operated. If the laboratory has new members, they can also register and add accounts for them to use the system, which is operated in the student management module.

\subsection{Device Category Management Module}

When the administrator enters this module, the category information of all devices will be displayed, and the administrator will add and modify the category information.

\subsection{Equipment Maintenance Management Module}

Only after the administrator confirms that the borrowing is successful, choose the equipment to submit the repair application to submit the repair application. After the application is submitted, the repair application status is still unprocessed. Student users can modify and delete the repair application to avoid misreporting. The repair application will be submitted to the device repair management module on the administrator page, and the administrator will review the repair information.

\subsection{Equipment Borrowing Management Module}

When a student user needs to borrow a device, select the device query module, you can query all borrowed device information and borrow, or you can choose the query condition to fuzzy query the device. After submitting the borrowing application, the administrator needs to review it. After the administrator processes the request, the borrowing is successful. The process of returning is similar to borrowing equipment.

\subsection{Borrowing Time Management Module}

When a user borrows a device, it is necessary to set the maximum number of borrowed devices for the number of devices borrowed by the user. After the user borrows, it is necessary to set that the user should return the device within the maximum borrowing time [8].

\subsection{System Usage}

After completing the system programming work, the teacher and students were organized to carry out a series of tests. After a long time of repeated tests, the ef- 
fectiveness and stability of the system were verified. At present, the system has been invested in the robot laboratory of our hospital use.

Because the computer used in the laboratory of our hospital is an internal network, I chose a computer to use as a server and set up servers in the background and the front desk to provide services 24 hours a day. The teacher in charge of management can log in to the management interface of any host in the network to manage the experimental equipment, while other teachers and ordinary students can also log in to the corresponding account in the network to apply for equipment and other tasks. The device management system is currently not correct. Entrance is provided for off-campus visits.

According to the feedback in actual use for a period of time, the originally designed functions of the system can basically be realized well. However, as administrator users and ordinary students have made some valuable comments. For example, the comments made by the administrator teacher hope that the real information of the teachers and students of the whole school can be imported into the database, which simplifies the input process during operation, and is not easy to enter errors, and can avoid the mixing of illegal users, to a certain extent, make the system more Safety; Some students have suggested that the current system equipment introduction is relatively simple. I hope that in the future improved version, the device information will be described in more detail, and various device instructions, tutorials, videos and other information will be included. Among them, it is convenient for teachers and students to further understand various devices. These opinions will be implemented in successively upgraded versions of the system.

\section{Conclusions}

Our robot lab equipment management system is the use of Java language, based on the SSM framework, using Eclipse project building tools to develop the management system, using MySQL to build a relational database, using Bootstrap and other technologies to build the front page. In the process of building the management system, the needs of the system are accurately grasped, and some programming problems are solved, new technology solutions are tried, and the system is integrated and operated well.

After a period of time, significantly improve the management level of laboratory equipment, solve the problem of laboratory equipment management confusion, maintenance and record inaccurate and inaccurate, equipment management inefficient, to achieve the unified and efficient management of all categories, for the future day-to-day operation provides a strong guarantee.

\section{Funding}

Supported by Hebei Province Education Department Hebei University Science and Technology Research Youth Fund Project (QN2017502); Hebei Higher Education Teaching Reform Research and Practice Project (2019GJJG483); Hebei Higher Education Teaching Research and Practice Project (2017GJJG261). 


\section{Conflicts of Interest}

The authors declare no conflicts of interest regarding the publication of this paper.

\section{References}

[1] Xu, J.X. (2019) Functional Design of Multimedia Equipment Management System in Colleges and Universities. Wuhan Business School, No. 10, 133-135.

[2] Yang, L. and Feng, T.T. (2019) Design and Research of Laboratory Equipment Management System Based on Java. School of Electronic Information and Electrical Engineering, 27, 54-56.

[3] Deng, Z.Q. and Liu, D.R. (2017) Design and Implementation of University Laboratory Management System Based on B/S. Journal of Beihua Institute of Aerospace Technology, 27, 54-56.

[4] Li, Y. (2016) Design and Implementation of SSM Framework in Web Application Development. Computer Technology and Development, 26, 190-194.

[5] Ya, J. (2016) Design and Implementation of Laboratory Management System. MA Thesis, Jilin University, Jilin.

[6] Zeng, H.Y. (2016) Design of Chemical Laboratory Management System. Electronic Technology and Software Engineering, No. 20, 61-62.

[7] Gu, W.W. (2016) Design and Implementation of Experimental Teaching Management System Based on J2EE Technology. MA Thesis, Beijing University of Posts and Telecommunications, Beijing.

[8] Zhang, Z.L. (2015) Research and Analysis of University Laboratory Information Management System. Yunnan University, No. 11, 93-94. 